of the substrate 'knock' on the spin of the atom and induce spin-reversals whereby the atom's spin and the spin of the substrate electrons become correlated, forming a singlet (that is, an antiparallel oriented) spin $\operatorname{state}^{6}$ (Fig. 1c). The experimenters in the group of Andreas Heinrich at IBM Almaden ${ }^{1}$ very carefully tuned the coupling between their single spin, a cobalt atom, and the copper substrate by introducing a thin insulating spacer layer of copper nitride, such that the resulting binding energy of the singlet state became smaller than the anisotropy barrier and also smaller than the splitting between the magnetic states at moderately high magnetic fields (up to 7 teslas). In this regime, they observed that the Kondo-assisted transport signatures were superimposed on those arising from spin-reversal associated with the IETS (the Kondo and IETS channels in Fig. 1c). The appearance of the Kondo effect in the experiment means that the cobalt atom is not a good 'magnetic bit', even at $0.5 \mathrm{~K}$, but rather that its magnetization flips roughly every 3 picoseconds, many orders of magnitude more often than the spin-flip induced by the tunnelling of electrons from the scanning tunnelling microscope tip. The surprising finding in the experiment by Otte et al. is that, despite this strong Kondo-related spin-flip, there is not a universal (isotropic) behaviour of the Kondo resonance in the system being studied. Instead, the Kondo resonance is strongly influenced by the crystalline anisotropy, providing valuable insight into the Kondo physics of such systems.

Even though many would consider the single Kondo impurity problem to be 'solved', such experiments at the atomic level show that the rich physics of such systems is far from being exhausted. There is still a lot of work to be done to reconcile the well-developed many-body theory of the Kondo effect with the realities of experimental systems, and it remains to be seen how, for example, atomic magnets on surfaces, such as those studied by Otte et al., can be successfully described by theory and calculations. Furthermore, the present experiment mimics the configuration of magnetic atoms in correlated electron materials. Thus, this physical insight may prove useful for the understanding of unusual effects in systems that display high-temperature and heavy-fermion superconductivity, colossal magnetoresistance or other strong electron-correlation effects.

\section{References}

1. Otte, A. F. et al. Nature Phys. 4, 847-850 (2008)

2. Gambardella, P. et al. Phys. Rev. Lett. 88, 047202 (2002).

3. Gambardella, P. et al. Science 300, 1130-1133 (2003).

4. Sessoli, R., Gatteschi, D., Caneschi, A. \& Novak, M. A. Nature 365, 141 (1993).

5. Hirjibehedin, C. F. et al. Science 317, 1199-1203 (2007).

6. Kouwenhoven, L. \& Glazman, L. Phys. World 14, 33-38 (January 2001).

\title{
ARTIFICIAL SPIN ICE
}

\section{Increasingly frustrated}
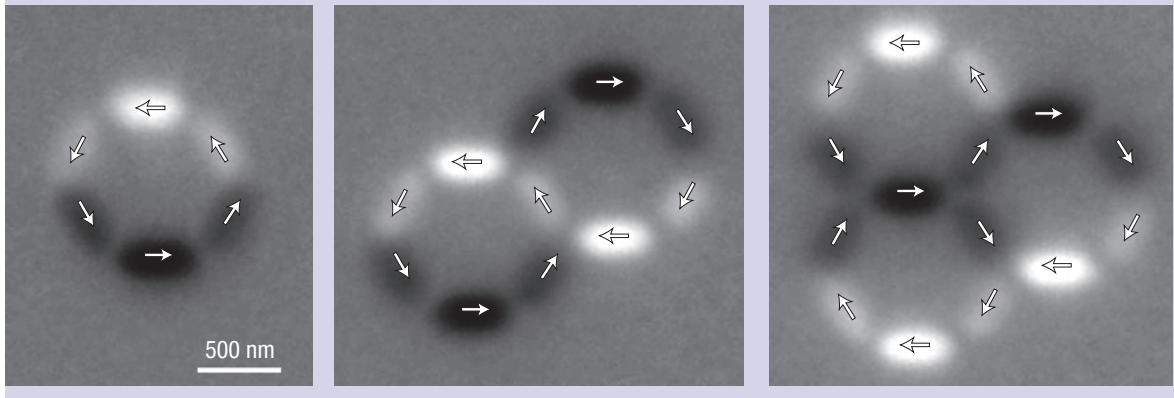

The properties of a large system can sometimes be elegantly rationalized by considering only simple concepts that govern the system as a whole; a deep understanding might, however, require details of how its smaller subsystems behave. A case in point is the study presented in Physical Review B by Elena Mengotti and colleagues, in which they look at the building blocks of an artificial spin-ice system and show how the characteristic behaviour of these systems emerges (Phys. Rev. B 78, 144402; 2008).

Spin ice describes a phase of magnetic systems in which, owing to geometric frustration, the ground state is disordered - and therefore possesses nonzero entropy - even when approaching zero temperature. A number of natural systems show such peculiar behaviour, but progress in lithographic patterning techniques has made it possible to create artificial spin ice, based on two-dimensional arrays of single-domain ferromagnetic islands. Following this line of work, Mengotti et al. have studied a kagome lattice, in which the magnetic islands form the edges of a honeycomb structure.

The magnetic moment of each island can be either parallel or antiparallel to its long axis, and the energetically most favourable configuration is one in which the magnetization vectors of neighbouring islands meet head-to-tail. That's fine for a single ring of islands, but not for two or more linked rings, because there are vertices at which three islands interact. At these points, the magnetic moments cannot be arranged such that each couple forms a head-to-tail pair - the system becomes 'frustrated'. The best compromise is to have two moments pointing towards the vertex, and one away from it, or vice versa. But for an extended honeycomb network, there are a huge number of equivalent arrangements that satisfy this twoin-one-out rule at each vertex, and, as a consequence, the ground state is disordered.

Mengotti and colleagues' interest is in the one- to three-ring building blocks of such networks. For these geometries, they calculated the energies of all possible magnetic configurations - which is possible for these systems, but becomes intractable for larger arrays - pinpointed the states with lowest energy and compared them with experimental observations. Following demagnetization of the sample, almost all of the single rings were found in the lowest-energy configuration, with all islands in a headto-tail configuration (as seen in the X-ray magnetic circular dichroism images here, in which brightness shows magnetization direction). In the two- and three-ring systems, however, frustration appears at the corners and for these geometries only about a half or a third of all samples, respectively, end up in the lowest-energy state. The implication is that, for a large network based on such building blocks, it might be practically impossible to reach the ground state.

Andreas Trabesinger 